\title{
Radiative lifetimes, branching fractions, and oscillator strengths of some levels in Be I
}

\author{
Xinghao Wang ${ }^{\mathrm{a}}$, Pascal Quinet ${ }^{\mathrm{b}, \mathrm{c}}$, Qiu Li ${ }^{\mathrm{a}}, \mathrm{Qi}$ Yu ${ }^{\mathrm{a}}$, Yongfan $\mathrm{Li}^{\mathrm{a}}$, Qian Wang ${ }^{\mathrm{d}}$, Yimin Gong ${ }^{\mathrm{a}, *}$, \\ Zhenwen Dai ${ }^{a, *}$ \\ ${ }^{a}$ Key Laboratory of Physics and Technology for Advanced Batteries (Ministry of Education), College of Physics, Jilin University, Changchun 130012, China \\ ${ }^{\mathrm{b}}$ Physique Atomique et Astrophysique, Université de Mons, Mons B-7000, Belgium \\ ${ }^{c}$ IPNAS, Université de Liège, Liège B-4000, Belgium \\ ${ }^{\mathrm{d}}$ College of Science, University of Science and Technology Liaoning, Anshan 114000, China
}

\section{A R T I C L E I N F O}

\section{Article history:}

Received 17 January 2018

Revised 23 March 2018

Accepted 23 March 2018

Available online 27 March 2018

\section{Keywords:}

Atomic spectroscopy

Radiative lifetime

Laser-induced fluorescence

\begin{abstract}
A B S T R A C T
Radiative lifetimes of five levels in Be I lying in the energy range 64,506.45-71,160.52 $\mathrm{cm}^{-1}$ were measured by the time-resolved laser-induced fluorescence technique. These new data, together with previously measured radiative lifetimes and two reliable calculated lifetimes, were combined with branching fractions obtained from pseudo-relativistic Hartree-Fock calculations to deduce semi-empirical transition probabilities and oscillator strengths for 90 Be I spectral lines involving upper levels ranging from $42,565.35$ to $72,251.27 \mathrm{~cm}^{-1}$.
\end{abstract}

() 2018 Elsevier Ltd. All rights reserved.

\section{Introduction}

Beryllium (Be, $Z=4$ ) has simple atomic structure entailing electronic interaction not only within the same shell, but also between different shells. Over the years, the four-electron Be I isoelectronic sequence has been taken as the testing ground for theoretical methods of calculating atomic structure and radiative properties, so the knowledge about beryllium atomic structure is important for studies of multi-electronic systems. Atomic radiative data such as lifetime and transition probability are helpful to gain an insight into the atomic structure. Spectroscopic data for Be I also have significant importance in astrophysics. For example, properties related to transition probabilities are needed for diagnostics of astrophysical plasmas [1,2]. One of the methods for determining transition probabilities and oscillator strengths is through the combination of radiative lifetimes with branching fractions (BFs). Hence, the lifetime measurements and determinations of BFs in Be I are of great significance.

In 1969, Bergström et al. measured seven lifetimes in Be I using a beam foil technique and presented transition probabilities for four lines from four of the seven levels [3]. With the same technique, radiative lifetime of the level $67,941.66 \mathrm{~cm}^{-1}$ was measured by Bromander et al. in 1969 [4]. In the same year, the

\footnotetext{
* Corresponding authors.

E-mail addresses: gongym@jlu.edu.cn (Y. Gong), dai@jlu.edu.cn (Z. Dai).
}

lifetimes of ten terms including two terms above the ionization limit 75,192.64 $\mathrm{cm}^{-1}$ were determined using the foil-excitation technique, and transition probabilities and oscillator strengths for seven transitions were also evaluated by Andersen et al. [5]. In 1971, Bromander revised eight lifetimes in Be I by a reinvestigation of the original decay curves from the experiments described in $[3,4]$ and obtained oscillator strengths for five transitions [6]. With the beam-foil technique, the lifetimes for the series $2 \mathrm{snd}{ }^{1} \mathrm{D}$ $(n=3-6)$ in Be I were measured, and oscillator strengths for the transitions down to $2 \mathrm{~s} 2 \mathrm{p}{ }^{1} \mathrm{P}^{\mathrm{o}}$ were determined by Andersen et al. in 1971 [7]. In the same year, Berry et al. measured the lifetime of the $2 \mathrm{p} 3 \mathrm{p}^{3} \mathrm{P}$ state using the beam-foil technique [8]. With the same technique, lifetimes of eight Be I terms, seven of which are located above the ionization limit, were determined by Hontzeas et al. in 1972 [9]. Lifetime of the level $68,780.86 \mathrm{~cm}^{-1}$ was measured by Poulsen et al. with fast-beam, zero-field level-crossing method in 1975 [10]. In 1980, selective laser excitation was used to measure lifetimes of the $2 \mathrm{~s} 3 \mathrm{~s}{ }^{3} \mathrm{~S}$ and $2 \mathrm{~s} 3 \mathrm{~d}{ }^{3} \mathrm{D}$ states of Be I by Kerkhoff et al. [11]. In 1994, Ellis et al. measured lifetimes of 2p3p and 2p3d levels in Be I and calculated them using the Hartree-Fock method [12]. Transition probabilities were reported for the $2 \mathrm{~s}^{2}{ }^{1} \mathrm{~S}-2 \mathrm{~s} 2 \mathrm{p}{ }^{1} \mathrm{p}^{\mathrm{o}}$ transition in Be I by Irving et al. and Schnabel and Kock in 1999 and 2000, respectively $[13,14]$.

In theoretical calculations, beryllium atom is also an attractive system because it provides interesting examples of electron correlation, core polarization, and relativistic effects [13]. In the past 70 years, there has been extensive theoretical research on transition 
probabilities of Be I. In 2010, Fuhr and Wiese carried out a comprehensive critical compilation of the atomic transition probabilities for the spectra of Be [15]. For other relevant papers, one can refer to the NIST bibliographic database [16].

The accuracy of theoretical results needs to be verified through comparison to experimental data. However, owing to the presence of highly-excited states and the small transition probabilities of electric dipole radiation between the singlet and triplet systems [11], the data reflecting beryllium atomic radiation properties are still incomplete and insufficiently accurate. In Be I radiative lifetimes of only 14 states below the ionization limit were measured, and also experimental transition probabilities or oscillator strengths of only 10 transitions were reported in literature. Extending this short list of available radiative parameter measurements would be valuable for benchmarking atomic structure calculations and for spectroscopic analysis of beryllium atom. In this paper, radiative lifetimes of five even-parity Be I levels below the ionization limit including two levels above $70,000 \mathrm{~cm}^{-1}$ were measured by time-resolved laser-induced fluorescence (TR-LIF) technique. For three of those levels, such experimental data are reported for the first time. In addition, we calculated the lifetimes and transition probabilities for all 17 Be I states, for which experimental lifetimes are known from literature and from the present work, using a theoretical model based on the pseudo-relativistic Hartree-Fock (HFR) method including core-polarization effects. By combining the available experimental data with calculated branching fractions, new semi-empirical transition probabilities and oscillator strengths were deduced for 90 spectral lines in atomic beryllium.

\section{Lifetime measurements}

In this paper, the radiative lifetimes are measured using the TR-LIF method. The experimental setup is the same as that described in a paper by our group [17], so only a brief outline is presented here. Ablation light emitted from a $532 \mathrm{~nm}$ Q-switched Nd: YAG laser with $8 \mathrm{~ns}$ pulse duration, $10 \mathrm{~Hz}$ repetition rate was focused on a Be target in a vacuum chamber to obtain free atoms. Then a beam of a dye laser (Sirah Cobra-stretch) operating with a 4-dicyanomethylene-2-methyl-6-pdimethylaminostyryl-4H-pyran (DCM) dye having a tunable range of $604-658 \mathrm{~nm}$ was sent through the vacuum chamber to selectively excite the atoms from metastable levels to the levels of interest. To obtain the needed excitation wavelength, one or two $\beta$-barium borate (BBO) type I crystals were employed to produce the second or third harmonics of the dye laser, and sometimes a hydrogen cell was also used to obtain different orders of Stokes and anti-Stokes components. The delay time between the excitation and ablation pulses was adjusted by a digital delay generator. Following the excitation, the fluorescence light detected by a photomultiplier tube (PMT, Hamamatsu R3896) in the direction perpendicular to the laser and the atomic beams was recorded and averaged in a $2.5 \mathrm{GHz}$ digital oscilloscope (Tektronix DPO7254). For each level, fluorescence curves were recorded at different delay times and more than 1000 shots were averaged to improve the signal-to-noise ratio of each curve recording.

In the measurement, all possible effects introducing systematic errors, such as collisional effects, radiation trapping, flight-out-ofview effect were minimized through appropriate variations of experimental conditions [18]. When the conditions are varied, e.g., reducing the excitation energy, adjusting the entrance slit position of the monochromator in vertical direction and the delay time between the excitation and ablation lasers, the measured values of lifetime oscillate around a constant within uncertainty indicating that influence of these systematic effects is negligibly small. With regard to the effect of the small Earth's magnetic field which may

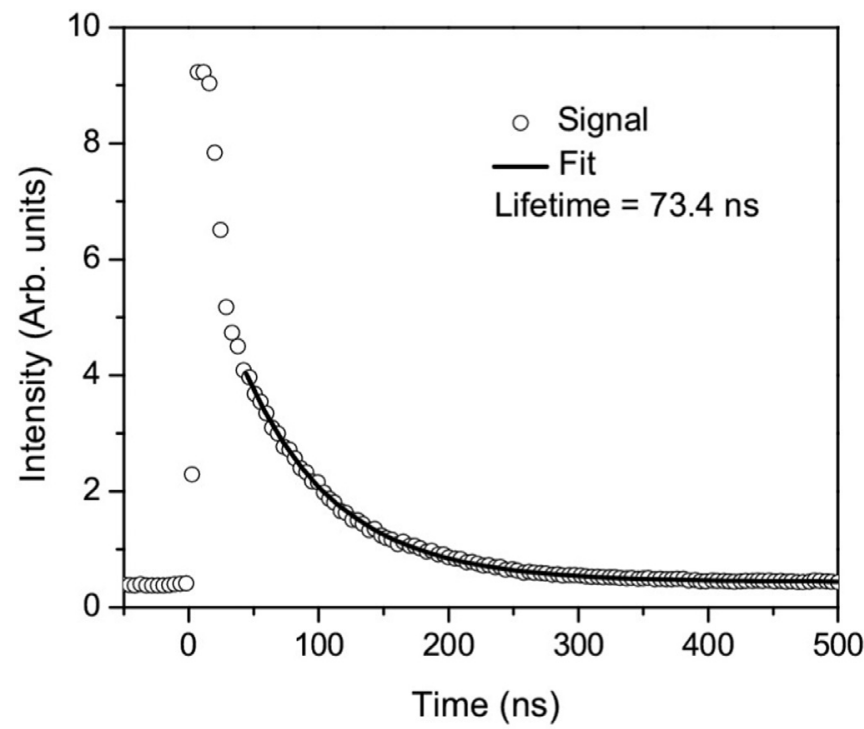

Fig. 1. A fluorescence decay curve of the $71,160.52 \mathrm{~cm}^{-1}$ level with an exponential fitting.

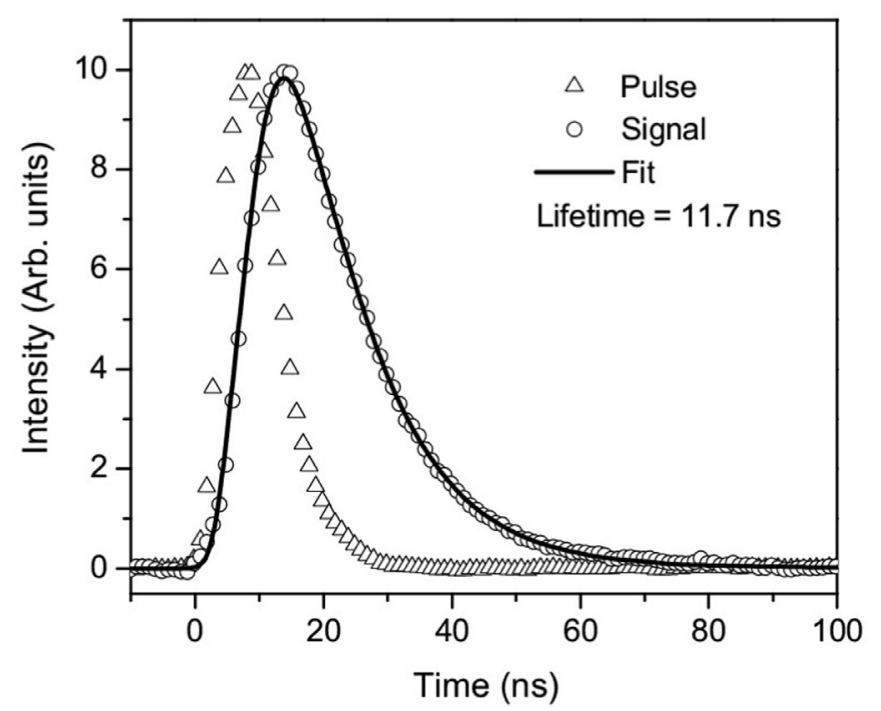

Fig. 2. A typical fluorescence decay curve of the $67,941.66 \mathrm{~cm}^{-1}$ level with the fitted curve convolving the laser pulse shape and an exponential.

cause Zeeman quantum beats, a static magnetic field of about 100 Gauss was employed, since the magnetic field strong enough will make the quantum beats too fast to be resolved by PMT owing to the limitation of response time. It was found that the applied magnetic field can also reduce the recombination background from the plasma [19].

The lifetime values longer than 40 ns were obtained by fitting the recorded fluorescence curve to an exponential function. For lifetimes shorter than $40 \mathrm{~ns}$, owing to limitation of the excitation pulse duration and the response time of detection system, the measurements were evaluated by fitting the fluorescence signal to the convolution of the detected laser pulse shape and an exponential function. As examples, a fluorescence curve of the $71,160.52 \mathrm{~cm}^{-1}$ level with an exponential fit is shown in Fig. 1, while the decay curve of the $67,941.66 \mathrm{~cm}^{-1}$ level together with a fitted convolution curve between the laser pulse and an exponential with a decay constant of $11.7 \mathrm{~ns}$ is given in Fig. 2 . 
Table 1

Measured and calculated lifetimes of Be I levels and comparison with previous results. The values marked in bold were used for deducing adjusted gA values by combining them with theoretical BFs.

\begin{tabular}{|c|c|c|c|c|c|c|c|c|}
\hline \multicolumn{4}{|c|}{ Upper level $^{\mathrm{a}}$} & \multirow[t]{3}{*}{$\lambda_{\text {Exc. }}(\mathrm{nm})$} & \multicolumn{4}{|c|}{ Lifetime (ns) } \\
\hline \multirow[t]{2}{*}{ Config. } & \multirow[t]{2}{*}{ Term } & \multirow[t]{2}{*}{$J$} & \multirow[t]{2}{*}{$E\left(\mathrm{~cm}^{-1}\right)$} & & \multicolumn{2}{|c|}{ This work } & \multicolumn{2}{|l|}{ Previous } \\
\hline & & & & & Exp. & Cal. & Exp. & Cal. \\
\hline $2 s 2 \mathrm{p}$ & ${ }^{1} \mathrm{P}^{\circ}$ & 1 & $42,565.35$ & & & 1.7 & $2.05(6)^{b}, 2.3(1)^{c}, 2.5(2)^{d}, 1.80(15)^{e}, 1.77(5)^{f}, \mathbf{1 . 8 5}(4)^{g}$ & $1.79^{\mathrm{h}}, 1.73^{\mathrm{i}}, 1.79^{\mathrm{k}}$ \\
\hline $2 \mathrm{~s} 3 \mathrm{~s}$ & ${ }^{3} S$ & 1 & $52,080.94$ & & & 6.7 & $5.95(16)^{b}, 7.3(2)^{\mathrm{c}}, 6.2(2)^{\mathrm{d}}, 6.4(2)^{1}$ & $6.70^{\mathrm{h}}, 6.65^{\mathrm{i}}, 6.61^{\mathrm{k}}$ \\
\hline \multirow{3}{*}{$2 \mathrm{p}^{2}$} & ${ }^{3} \mathrm{P}$ & 0 & $59,693.65$ & & & 2.2 & 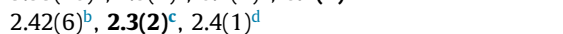 & $2.31^{\mathrm{h}}, 2.37^{\mathrm{k}}$ \\
\hline & & 1 & $59,695.07$ & & & 2.2 & 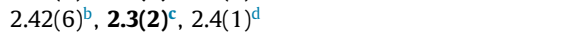 & $2.31^{\mathrm{h}}, 2.37^{\mathrm{k}}$ \\
\hline & & 2 & $59,697.08$ & & & 2.2 & $2.42(6)^{b}, 2.3(2)^{c}, 2.4(1)^{d}$ & $2.31^{\mathrm{h}}, 2.37^{\mathrm{k}}$ \\
\hline $2 \mathrm{~s} 3 \mathrm{~d}$ & ${ }^{3} \mathrm{D}$ & $1,2,3$ & $62,053.72$ & & & 5.6 & $5.00(14)^{b}, 4.9(2)^{c}, 5.4(2)^{d}, \mathbf{5 . 2}(2)^{l}$ & $5.18^{\mathrm{h}}, 5.26^{\mathrm{i}}, 5.14^{\mathrm{k}}$ \\
\hline $2 \mathrm{~s} 3 \mathrm{~d}$ & ${ }^{1} \mathrm{D}$ & 2 & $64,428.31$ & & & 10.6 & $12.0(3)^{b}, 10.0(2)^{c}, \mathbf{1 2 . 3}(\mathbf{4})^{d}, 10.4(10)^{m}$ & $12.3^{\mathrm{h}}, 12.2^{\mathrm{i}}, 12.5^{\mathrm{k}}$ \\
\hline $2 \mathrm{~s} 4 \mathrm{~s}$ & ${ }^{3} \mathrm{~S}$ & 1 & $64,506.45$ & 235.066 & 18.2(7) & 19.8 & & $17.9^{\mathrm{i}}$ \\
\hline 2s4d & ${ }^{3} \mathrm{D}$ & 1 & $67,941.66$ & 217.496 & 11.7(6) & 14.6 & $11.5(5)^{c}, 10.8(4)^{d}, 11.2(6)^{n}$ & $11.7^{\mathrm{h}}, 11.73^{\mathrm{i}}$ \\
\hline $2 s 4 d$ & ${ }^{1} \mathrm{D}$ & 2 & $68,780.86$ & & & 15.4 & $18.8(5)^{b}, 13.6(2)^{c}, \mathbf{1 9 . 3}(5)^{d}, 14.0(15)^{m}, 14.5(10)^{o}$ & $19.9^{\mathrm{h}}, 20.1^{\mathrm{i}}$ \\
\hline $2 \mathrm{~s} 5 \mathrm{~s}$ & ${ }^{3} \mathrm{~S}$ & 1 & $69,010.20$ & 212.554 & $38.6(6)$ & 42.6 & $10.5(5)^{\mathrm{c}}$ & $38.4^{\mathrm{h}}, 38.4^{\mathrm{i}}$ \\
\hline $2 \mathrm{~s} 5 \mathrm{~d}$ & ${ }^{3} \mathrm{D}$ & 1 & $70,603.76$ & 205.588 & $23.3(10)$ & 30.1 & & $22.5^{\mathrm{i}}$ \\
\hline $2 \mathrm{~s} 5 \mathrm{~d}$ & ${ }^{1} \mathrm{D}$ & 2 & $71,002.34$ & & & 24.4 & $19(2)^{\mathrm{m}}$ & $\mathbf{3 5 . 1}^{\mathrm{h}}, 35.5^{\mathrm{i}}$ \\
\hline $2 s 6 s$ & ${ }^{3} \mathrm{~S}$ & 1 & $71,160.52$ & 203.260 & 73.4(27) & 79.4 & & $69.6^{\mathrm{i}}$ \\
\hline $2 \mathrm{~s} 6 \mathrm{~d}$ & ${ }^{1} \mathrm{D}$ & 2 & $72,251.27$ & & & 36.8 & $38(4)^{\mathrm{m}}$ & 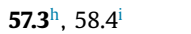 \\
\hline
\end{tabular}

a Kramida and Martin [24].

b Bergström et al. [3] by non-selective excitation (NSE).

c Andersen et al. [5] by NSE.

d Bromander [6] by NSE.

e Hontzeas et al. [9] by NSE.

f Irving et al[13]. by NSE.

g Schnabel and Kock [14] by selective excitation (SE).

h Moccia and Spizzo [26].

i Chang [27].

$\mathrm{k}$ Tachiev and Froese Fischer [29].

${ }^{1}$ Kerkhoff et al. [11] by SE.

$m$ Andersen et al [7] by NSE.

${ }^{n}$ Bromander et al. [4] by NSE.

o Poulsen et al. [10] by NSE.

\section{Calculations of lifetimes and oscillator strengths}

The computational approach considered in the present work is the pseudo-relativistic Hartree-Fock (HFR) method [20] in which we have incorporated the core-polarization effects, in frames of the so-called HFR + CPOL method (see e.g. $[21,22]$ ). The configuration sets retained for the calculations were $1 s^{2} 2 s^{2}, 1 s^{2} 2 s n s(n=3-10)$, $1 \mathrm{~s}^{2} 2 \mathrm{snd}(n=3-10), 1 \mathrm{~s}^{2} 2 \mathrm{p}^{2}, 1 \mathrm{~s}^{2} 2 \mathrm{p} n \mathrm{p}(n=3-10)$ and $1 \mathrm{~s}^{2} 2 \mathrm{p} n \mathrm{f}(n=4-$ $10)$ for the even parity, and $1 \mathrm{~s}^{2} 2 \mathrm{snp}(n=3-10), 1 \mathrm{~s}^{2} 2 \mathrm{snf}(n=4-10)$, $1 \mathrm{~s}^{2} 2 \mathrm{p} n \mathrm{~s}(n=3-10)$ and $1 \mathrm{~s}^{2} 2 \mathrm{p} n \mathrm{~d}(n=3-10)$ for the odd parity.

The estimate of the core-polarization contributions requires the knowledge of the dipole polarizability of the ionic core, $\alpha_{d}$, and of the cut-off radius, $r_{c}$. For the first parameter, we used the value of the static dipole polarizability computed by Johnson et al. [23] for Be III, i.e. $\alpha_{d}=0.05 a_{0}{ }^{3}$. The cut-off radius, $r_{c}$, was chosen equal to $0.41 a_{0}$, which corresponds to the HFR average value $<r>$ of the core orbital $1 \mathrm{~s}$.

In addition, the radial energy parameters, such as average energies, Slater integrals and spin-orbit parameters, were adjusted with a least-squares optimization program minimizing the discrepancies between the calculated and the available experimental energy levels taken from the NIST compilation [24,25] for the configurations of the type $1 s^{2} 2 s^{2}, 1 s^{2} 2 p^{2}, 1 s^{2} 2 s n s, 1 s^{2} 2 s n p, 1 s^{2} 2 s n d$ and $1 s^{2} 2 s n f$.

\section{Results and discussion}

The lifetimes measured in this paper for 5 levels in Be I are listed in Table 1, where all other 12 levels for which experimental lifetimes are known in literature are also presented. Furthermore, calculated lifetimes for the 17 Be I levels along with previously reported results are also shown in the table for comparison. The energies and classifications of the levels in the table are from the compilation by Kramida and Martin [24]. The measurement uncer- tainties consist of possible remaining systematic errors and statistical errors from different recordings.

For the two levels $67,941.66$ and $69,010.2 \mathrm{~cm}^{-1}$, the lifetimes were measured both by others and by us. For the first one, the results of this work are consistent with literature. For the second one, there is a discrepancy of up to $72.8 \%$ ((ours - previous)/ours) with the experimental result from [5], but our measurement result agrees well with our calculations and those from [26,27]. Considering the reliability of the calculated lifetime results (see below), our measured value of $38.6 \mathrm{~ns}$ for the $69,010.2 \mathrm{~cm}^{-1}$ level seems more reliable than $10.5 \mathrm{~ns}$ from [5].

As for the comparison between our experimental and calculated lifetime results, their differences for the five levels $(64,506.45$, $67,941.66,69,010.20,70,603.76$ and $71,160.52 \mathrm{~cm}^{-1}$ ) are $8.8 \%, 24.8 \%$, $10.4 \%, 29.2 \%$ and $8.2 \%$, respectively, so we can say that they are in fair agreement. In comparison, the calculation results by Moccia and Spizzo [26] and by Chang [27] agree somewhat better with our experimental results than those calculated in this paper. However, the measured lifetimes of the 71,002.34 and $72,251.27 \mathrm{~cm}^{-1}$ levels are much closer to our calculations than to those of other authors. For clarity, Fig. 3 shows a comparison between our calculation results and those from Refs. [26,27] along with all the experimental lifetime data.

Table 2 presents the HFR + CPOL theoretical BFs, transition probabilities $g A$ and oscillator strengths on a logarithmic scale $\log (g f)$ obtained from $g A$ values using the experimental wavenumbers for 90 transitions with the 17 levels listed in Table 1 as upper levels. In Tables 1 and 2, the wavelengths calculated from the energy levels taken from the NIST ASD [25] are given in standard air. Conversion from vacuum to air was made using the five-parameter formula from Peck and Reeder [28]. Previously published semiempirical $\log (g f)$ results using the weakest bound electron potential model theory (WBEPMT) and the quantum defect orbital (QDO) 
Table 2

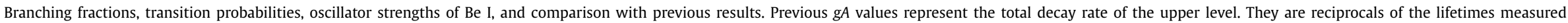
in the quoted works.

\begin{tabular}{|c|c|c|c|c|c|c|c|c|c|c|c|c|c|c|}
\hline \multicolumn{2}{|c|}{ Upper level $^{\mathrm{a}}$} & \multicolumn{2}{|c|}{ Lower level $^{\mathrm{a}}$} & \multirow[t]{3}{*}{$\lambda_{\text {air }}(\mathrm{nm})$} & \multirow[t]{3}{*}{$\mathrm{BF}$} & \multicolumn{3}{|c|}{$g A\left(10^{6} \mathrm{~s}^{-1}\right)$} & \multicolumn{6}{|c|}{$\log (g f)$} \\
\hline \multirow[t]{2}{*}{ Assign. } & \multirow[t]{2}{*}{$\begin{array}{l}E\left(\mathrm{~cm}^{-1}\right) \\
\text { Lifetime (ns) }\end{array}$} & \multirow[t]{2}{*}{ Assign. } & \multirow[t]{2}{*}{$E\left(\mathrm{~cm}^{-1}\right)$} & & & \multicolumn{2}{|l|}{ This work } & \multirow{2}{*}{$\begin{array}{l}\text { Previous } \\
\text { Exp. }\end{array}$} & \multicolumn{2}{|c|}{ This work } & \multicolumn{4}{|l|}{ Previous $^{\mathrm{b}}$} \\
\hline & & & & & & HFR & Adjusted $^{\mathrm{n}}$ & & $\overline{\text { HFR }}$ & Adjusted $^{\mathrm{n}}$ & WBEPMT & QDO & MCHF & Exp. \\
\hline $2 s 2 \mathrm{p}^{1} \mathrm{P}_{1}^{\circ}$ & $\begin{array}{l}42,565.35 \\
\tau=1.85(4)^{c}\end{array}$ & $2 \mathrm{~s}^{21} \mathrm{~S}_{0}$ & 0.00 & 234.861 & 1 & 1720 & 1622(B+) & $1463(46)^{\mathrm{h}}, 1200^{\mathrm{e}}$ & 0.15 & $0.13(\mathrm{~B}+)$ & & & 0.14 & $0.15^{\mathrm{i}}, 0.13^{\mathrm{c}}$ \\
\hline \multirow[t]{2}{*}{$2 \mathrm{~s} 3 \mathrm{~s}^{3} \mathrm{~S}_{1}$} & $\begin{array}{l}52,080.94 \\
\tau=6.4(2)^{d}\end{array}$ & $\begin{array}{l}2 \mathrm{~s} 2 \mathrm{p}^{3} \mathrm{P}_{0}^{\circ} \\
2 \mathrm{~s} 2 \mathrm{p}^{3} \mathrm{P}_{1}^{\circ}\end{array}$ & $\begin{array}{l}21,978.28 \\
21,978.925\end{array}$ & $\begin{array}{l}332.101 \\
332.108\end{array}$ & $\begin{array}{l}0.111 \\
0.333\end{array}$ & $\begin{array}{l}50.0 \\
150\end{array}$ & $\begin{array}{l}52.1(\mathrm{C}+) \\
156(\mathrm{C}+)\end{array}$ & $504(18)^{\mathrm{h}}, 411^{\mathrm{e}}$ & $\begin{array}{l}-1.08 \\
-0.61\end{array}$ & $\begin{array}{l}-1.06(\mathrm{C}+) \\
-0.59(\mathrm{C}+)\end{array}$ & $\begin{array}{l}-1.17 \\
-0.69\end{array}$ & $\begin{array}{l}-1.01 \\
-0.54\end{array}$ & $\begin{array}{l}-1.07 \\
-0.60\end{array}$ & \\
\hline & & $2 \mathrm{~s} 2 \mathrm{p}^{3} \mathrm{P}_{2}{ }^{\circ}$ & $21,981.27$ & 332.134 & 0.556 & 250 & $260(B)$ & & -0.38 & $-0.37(\mathrm{~B})$ & -0.47 & -0.32 & -0.38 & \\
\hline $2 \mathrm{p}^{23} \mathrm{P}_{0}$ & $\begin{array}{l}59,693.65 \\
\tau=2.3(2)^{\mathrm{e}}\end{array}$ & $2 \mathrm{~s} 2 \mathrm{p}^{3} \mathrm{P}_{1}^{\circ}$ & $21,978.925$ & 265.070 & 1.00 & 456 & $435(\mathrm{~B}+)$ & $413(12)^{\mathrm{h}}, 435^{\mathrm{e}}$ & -0.32 & $-0.34(\mathrm{~B}+)$ & & & -0.35 & \\
\hline \multirow[t]{3}{*}{$2 \mathrm{p}^{2}{ }^{3} \mathrm{P}_{1}$} & $59,695.07$ & $2 \mathrm{~s} 2 \mathrm{p}^{3} \mathrm{P}_{0}{ }^{\circ}$ & $21,978.28$ & 265.055 & 0.333 & 456 & $434(\mathrm{~B}+)$ & & -0.32 & $-0.34(\mathrm{~B}+)$ & & & -0.35 & \\
\hline & $\tau=2.3(2)^{e}$ & $2 \mathrm{~s} 2 \mathrm{p}^{3} \mathrm{P}_{1}{ }^{\circ}$ & $21,978.925$ & 265.061 & 0.250 & 342 & $326(\mathrm{~B}+)$ & & -0.44 & $-0.46(\mathrm{~B}+)$ & & & -0.48 & \\
\hline & & $2 \mathrm{~s} 2 \mathrm{p}^{3} \mathrm{P}_{2}{ }^{\circ}$ & $21,981.27$ & 265.076 & 0.417 & 570 & $544(\mathrm{~B}+)$ & & -0.22 & $-0.24(\mathrm{~B}+)$ & & & -0.25 & \\
\hline \multirow[t]{2}{*}{$2 \mathrm{p}^{23} \mathrm{P}_{2}$} & $59,697.08$ & $2 \mathrm{~s} 2 \mathrm{p}^{3} \mathrm{P}_{1}{ }^{\circ}$ & $21,978.925$ & 265.045 & 0.250 & 570 & $543(\mathrm{~B}+)$ & & -0.22 & $-0.24(\mathrm{~B}+)$ & & & -0.25 & \\
\hline & $\tau=2.3(2)^{\mathrm{e}}$ & $2 \mathrm{~s} 2 \mathrm{p}^{3} \mathrm{P}_{2}{ }^{\circ}$ & $21,981.27$ & 265.062 & 0.750 & 1711 & $1630(\mathrm{~B}+)$ & & 0.26 & $0.23(\mathrm{~B}+)$ & & & 0.22 & \\
\hline \multirow[t]{6}{*}{$2 \mathrm{~s} 3 \mathrm{~d}^{3} \mathrm{D}_{1}$} & $62,053.72$ & $2 \mathrm{~s} 2 \mathrm{p}^{3} \mathrm{P}_{0}{ }^{\circ}$ & $21,978.28$ & 249.454 & 0.550 & 293 & $317(\mathrm{~B})$ & $612^{\mathrm{e}}$ & -0.56 & $-0.53(\mathrm{~B})$ & -0.58 & -0.59 & -0.53 & \\
\hline & $\tau=5.2(2)^{\mathrm{d}}$ & $2 \mathrm{~s} 2 \mathrm{p}^{3} \mathrm{P}_{1}{ }^{\circ}$ & $21,978.925$ & 249.458 & 0.413 & 220 & $238(\mathrm{C}+)$ & & -0.69 & $-0.65(\mathrm{C}+)$ & -0.70 & -0.71 & -0.65 & \\
\hline & & $2 \mathrm{~s} 2 \mathrm{p}^{3} \mathrm{P}_{2}{ }^{\circ}$ & $21,981.27$ & 249.473 & 0.028 & 14.7 & $15.9(\mathrm{C})$ & & -1.86 & $-1.83(\mathrm{C})$ & -1.88 & -1.89 & -1.83 & \\
\hline & & $2 \mathrm{~s} 3 \mathrm{p}^{3} \mathrm{P}^{\mathrm{o}}{ }_{0}$ & $58,907.45$ & 3177.500 & 0.005 & 2.83 & $2.30(\mathrm{E})$ & & -0.37 & $-0.33(\mathrm{E})$ & -0.28 & -0.25 & -0.30 & \\
\hline & & $2 \mathrm{~s} 3 \mathrm{p}^{3} \mathrm{P}^{\mathrm{o}}{ }_{1}$ & $58,907.45$ & 3177.500 & 0.004 & 2.12 & $3.06(E)$ & & -0.49 & $-0.46(\mathrm{E})$ & -0.41 & -0.38 & -0.43 & \\
\hline & & $2 \mathrm{~s} 3 \mathrm{p}^{3} \mathrm{P}_{2}{ }^{\circ}$ & $58,907.83$ & 3177.884 & 0.0003 & 0.141 & $0.152(\mathrm{E})$ & & -1.67 & $-1.64(\mathrm{E})$ & -1.58 & -1.53 & -1.60 & \\
\hline \multirow{4}{*}{$2 \mathrm{~s} 3 \mathrm{~d}^{3} \mathrm{D}_{2}$} & $62,053.72$ & $2 \mathrm{~s} 2 \mathrm{p}^{3} \mathrm{P}_{1}{ }^{\circ}$ & $21,978.925$ & 249.458 & 0.743 & 659 & $714(\mathrm{~B}+)$ & $1020^{e}$ & -0.21 & $-0.18(\mathrm{~B}+)$ & -0.23 & -0.23 & -0.18 & \\
\hline & $\tau=5.2(2)^{d}$ & $2 \mathrm{~s} 2 \mathrm{p}^{3} \mathrm{P}_{2}{ }^{\circ}$ & $21,981.27$ & 249.473 & 0.248 & 220 & $238(\mathrm{C}+)$ & & -0.69 & $-0.65(\mathrm{C}+)$ & -0.70 & -0.71 & -0.65 & \\
\hline & & $2 \mathrm{~s} 3 \mathrm{p}^{3} \mathrm{P}_{1}{ }^{\circ}$ & $58,907.45$ & 3177.500 & 0.007 & 6.36 & $6.89(\mathrm{E})$ & & -0.02 & $0.018(\mathrm{E})$ & 0.07 & 0.10 & 0.05 & \\
\hline & & $2 \mathrm{~s} 3 \mathrm{p}^{3} \mathrm{P}_{2}{ }^{\circ}$ & $58,907.83$ & 3177.884 & 0.002 & 2.12 & $2.30(\mathrm{E})$ & & -0.49 & $-0.45(\mathrm{E})$ & -0.41 & -0.38 & -0.43 & \\
\hline \multirow[t]{2}{*}{$2 \mathrm{~s} 3 \mathrm{~d}^{3} \mathrm{D}_{3}$} & $62,053.72$ & $2 \mathrm{~s} 2 \mathrm{p}^{3} \mathrm{P}_{2}{ }^{\circ}$ & $21,981.27$ & 249.473 & 0.990 & 1230 & 1333(B+) & $1428^{e}$ & 0.06 & $0.09(\mathrm{~B}+)$ & 0.05 & 0.04 & 0.09 & \\
\hline & $\tau=5.2(2)^{\mathrm{d}}$ & $2 \mathrm{~s} 3 \mathrm{p}^{3} \mathrm{P}_{2}{ }^{\circ}$ & $58,907.83$ & 3177.884 & 0.010 & 11.9 & 12.9(E) & & 0.26 & $0.29(\mathrm{E})$ & 0.34 & 0.37 & 0.32 & \\
\hline \multirow{2}{*}{$2 \mathrm{~s} 3 \mathrm{~d}{ }^{1} \mathrm{D}_{2}$} & $64,428.31$ & $2 \mathrm{~s} 2 \mathrm{p}^{1} \mathrm{P}_{1}^{\circ}$ & $42,565.35$ & 457.266 & 0.935 & 439 & $380(\mathrm{~B}+)$ & $500^{e}$ & 0.14 & $0.08(\mathrm{~B}+)$ & 0.01 & 0.14 & 0.09 & $0.19^{\mathrm{e}}, 0.18^{\mathrm{k}}$ \\
\hline & $\tau=12.3(4)^{\mathrm{f}}$ & $2 \mathrm{~s} 3 \mathrm{p}^{1} \mathrm{P}_{1}{ }^{\circ}$ & $60,187.34$ & 2357.308 & 0.065 & 30.3 & $26.2(\mathrm{C}+)$ & & 0.40 & $0.34(\mathrm{C}+)$ & 0.41 & 0.35 & 0.31 & \\
\hline $2 s 4 s^{3} S_{1}$ & $64,506.45$ & $2 \mathrm{~s} 2 \mathrm{p}^{3} \mathrm{P}_{0}{ }^{\circ}$ & $21,978.28$ & 235.066 & 0.085 & 12.9 & $14.0(C+)$ & & -1.97 & $-1.94(\mathrm{C}+)$ & -1.94 & -1.86 & -1.94 & \\
\hline & $\tau=18.2(7)$ & $2 \mathrm{~s} 2 \mathrm{p}^{3} \mathrm{P}_{1}{ }^{\circ}$ & $21,978.925$ & 235.070 & 0.254 & 38.6 & $41.9(\mathrm{C}+)$ & & -1.50 & $-1.46(\mathrm{C}+)$ & -1.47 & -1.38 & -1.46 & \\
\hline & & $2 \mathrm{~s} 2 \mathrm{p}^{3} \mathrm{P}_{2}{ }^{\circ}$ & $21,981.27$ & 235.083 & 0.424 & 64.3 & $69.8(\mathrm{C}+)$ & & -1.27 & $-1.24(\mathrm{C}+)$ & -1.24 & -1.16 & -1.24 & \\
\hline & & $2 \mathrm{~s} 3 \mathrm{p}^{3} \mathrm{P}_{0}{ }^{\circ}$ & $58,907.45$ & 1785.546 & 0.026 & 4.00 & $4.30(\mathrm{C})$ & & -0.72 & $-0.68(\mathrm{C})$ & -0.66 & -0.59 & -0.67 & \\
\hline & & $2 \mathrm{~s} 3 \mathrm{p}^{3} \mathrm{P}_{1}{ }^{\circ}$ & $58,907.45$ & 1785.546 & 0.079 & 12.0 & $13.0(\mathrm{C}+)$ & & -0.24 & $-0.21(\mathrm{C}+)$ & -0.18 & -0.11 & -0.19 & \\
\hline & & $2 s 3 p^{3} \mathrm{P}_{2}{ }^{\circ}$ & $58,907.83$ & 1785.667 & 0.132 & 20.0 & $21.7(\mathrm{C}+)$ & & -0.02 & $0.016(\mathrm{C}+)$ & 0.04 & 0.11 & 0.03 & \\
\hline $2 \mathrm{~s} 4 \mathrm{~d}^{3} \mathrm{D}_{1}$ & $67,941.66$ & $2 \mathrm{~s} 2 \mathrm{p}^{3} \mathrm{P}_{0}{ }^{\circ}$ & $21,978.28$ & 217.496 & 0.520 & 107 & 133(B) & & -1.12 & $-1.02(\mathrm{~B})$ & -1.03 & -1.05 & -1.02 & \\
\hline & $\tau=11.7(6)$ & $2 \mathrm{~s} 2 \mathrm{p}^{3} \mathrm{P}_{1}{ }^{\circ}$ & $21,978.925$ & 217.499 & 0.390 & 80.2 & $100(C+)$ & & -1.24 & $-1.15(\mathrm{C}+)$ & -1.16 & -1.18 & -1.14 & \\
\hline & & $2 \mathrm{~s} 2 \mathrm{p}^{3} \mathrm{P}_{2}{ }^{\circ}$ & $21,981.27$ & 217.510 & 0.026 & 5.35 & $6.67(\mathrm{C})$ & & -2.42 & $-2.32(\mathrm{C})$ & -2.33 & -2.35 & -2.32 & \\
\hline & & $2 \mathrm{~s} 3 \mathrm{p}^{3} \mathrm{P}_{0}{ }^{\circ}$ & $58,907.45$ & 1106.601 & 0.033 & 6.74 & $8.40(\mathrm{C})$ & & -0.90 & $-0.81(\mathrm{C})$ & -0.99 & -0.86 & -0.88 & \\
\hline & & $2 \mathrm{~s} 3 \mathrm{p}^{3} \mathrm{P}_{1}{ }^{\circ}$ & $58,907.45$ & 1106.601 & 0.025 & 5.06 & $6.31(\mathrm{C})$ & & -1.02 & $-0.936(\mathrm{C})$ & -1.11 & -0.98 & -1.0 & \\
\hline & & $2 \mathrm{~s} 3 \mathrm{p}^{3} \mathrm{P}_{2}{ }^{\circ}$ & $58,907.83$ & 1106.647 & 0.002 & 0.337 & $0.420(\mathrm{E})$ & & -2.20 & $-2.11(\mathrm{E})$ & -2.29 & -2.16 & -2.18 & \\
\hline & & $2 \mathrm{~s} 4 \mathrm{p}^{3} \mathrm{P}_{0}{ }^{\circ}$ & $66,811.88$ & 8848.868 & 0.003 & 0.530 & $0.660(\mathrm{E})$ & & -0.21 & $-0.11(\mathrm{E})$ & -0.06 & -0.07 & -0.09 & \\
\hline & & $2 \mathrm{~s} 4 \mathrm{p}^{3} \mathrm{P}_{1}^{\circ}$ & $66,811.88$ & 8848.868 & 0.002 & 0.400 & $0.500(\mathrm{E})$ & & -0.33 & $-0.24(\mathrm{E})$ & -0.18 & -0.19 & -0.22 & \\
\hline & & $2 s 4 \mathrm{p}^{3} \mathrm{P}_{2}{ }^{\circ}$ & $66,811.88$ & 8848.868 & 0.0001 & 0.0260 & $0.0330(\mathrm{E})$ & & -1.51 & $-1.41(\mathrm{E})$ & & & & \\
\hline
\end{tabular}


Table 2 (continued)

\begin{tabular}{|c|c|c|c|c|c|c|c|c|c|c|c|c|c|c|}
\hline \multicolumn{2}{|c|}{ Upper level $^{\mathrm{a}}$} & \multicolumn{2}{|c|}{ Lower level $^{\mathrm{a}}$} & \multirow[t]{3}{*}{$\lambda_{\text {air }}(\mathrm{nm})$} & \multirow[t]{3}{*}{$\mathrm{BF}$} & \multicolumn{3}{|c|}{$g A\left(10^{6} \mathrm{~s}^{-1}\right)$} & \multicolumn{6}{|c|}{$\log (g f)$} \\
\hline \multirow[t]{2}{*}{ Assign. } & \multirow{2}{*}{$\begin{array}{l}E\left(\mathrm{~cm}^{-1}\right) \\
\text { Lifetime (ns) }\end{array}$} & \multirow[t]{2}{*}{ Assign. } & \multirow[t]{2}{*}{$E\left(\mathrm{~cm}^{-1}\right)$} & & & \multicolumn{2}{|c|}{ This work } & \multirow{2}{*}{$\begin{array}{l}\text { Previous } \\
\text { Exp. }\end{array}$} & \multicolumn{2}{|c|}{ This work } & \multicolumn{4}{|l|}{ Previous $^{\mathrm{b}}$} \\
\hline & & & & & & HFR & Adjusted $^{\mathrm{n}}$ & & $\overline{\text { HFR }}$ & Adjusted $^{\mathrm{n}}$ & WBEPMT & QDO & MCHF & Exp. \\
\hline \multirow[t]{4}{*}{$2 \mathrm{~s} 4 \mathrm{~d}^{1}{ }^{1} \mathrm{D}_{2}$} & $68,780.86$ & $2 \mathrm{~s} 2 \mathrm{p}^{1} \mathrm{P}_{1}^{\circ}$ & $42,565.35$ & 381.345 & 0.968 & 313 & $251(B+)$ & $370^{e}$ & -0.17 & $-0.26(\mathrm{~B}+)$ & -0.31 & -0.37 & -0.28 & $-0.09^{e},-0.12^{k}$ \\
\hline & $\tau=19.3(5)^{\mathrm{f}}$ & $2 \mathrm{~s} 3 \mathrm{p}^{1} \mathrm{P}_{1}{ }^{\circ}$ & $60,187.34$ & 1163.349 & 0.003 & 0.94 & $0.75(\mathrm{E})$ & & -1.72 & $-1.82(\mathrm{E})$ & -1.52 & -0.78 & -1.28 & \\
\hline & & $2 s 4 p^{1} \mathrm{P}_{1}^{\circ}$ & $67,034.70$ & 5725.291 & 0.027 & 8.87 & 7.11(C) & & 0.64 & $0.54(\mathrm{C})$ & 0.59 & 0.62 & 0.56 & \\
\hline & & $2 s 4 \mathrm{f}^{1} \mathrm{~F}_{3}^{\circ}$ & $68,241.18$ & $18,524.449$ & 0.0015 & 0.473 & $0.38(\mathrm{E})$ & & 0.39 & $0.29(\mathrm{E})$ & & & & \\
\hline \multirow[t]{9}{*}{$2 s 5 s^{3} S_{1}$} & $69,010.20$ & $2 \mathrm{~s} 2 \mathrm{p}^{3} \mathrm{P}_{0}{ }^{\circ}$ & $21,978.28$ & 212.554 & 0.076 & 5.32 & $5.87(\mathrm{D}+)$ & & -2.44 & $-2.40(\mathrm{D}+)$ & -2.38 & -2.30 & -2.39 & \\
\hline & $\tau=38.6(6)$ & $2 \mathrm{~s} 2 \mathrm{p}^{3} \mathrm{P}^{\mathrm{o}}{ }_{1}$ & $21,978.925$ & 212.557 & 0.227 & 16.0 & $17.7(\mathrm{D}+)$ & & -1.97 & $-1.92(\mathrm{D}+)$ & -1.90 & -1.83 & -1.91 & \\
\hline & & $2 \mathrm{~s} 2 \mathrm{p}^{3} \mathrm{P}_{2}{ }^{\circ}$ & $21,981.27$ & 212.568 & 0.378 & 26.6 & $29.4(\mathrm{D}+)$ & & -1.74 & $-1.70(\mathrm{D}+)$ & -1.68 & -1.61 & -1.69 & \\
\hline & & $2 \mathrm{~s} 3 \mathrm{p}^{3} \mathrm{P}_{0}{ }^{\circ}$ & $58,907.45$ & 989.558 & 0.021 & 1.49 & $1.65(\mathrm{D}+)$ & & -1.66 & $-1.62(\mathrm{D}+)$ & -1.64 & -1.57 & -1.63 & \\
\hline & & $2 \mathrm{~s} 3 \mathrm{p}^{3} \mathrm{P}_{1}^{\circ}$ & $58,907.45$ & 989.558 & 0.064 & 4.47 & $4.94(\mathrm{C})$ & & -1.18 & $-1.14(\mathrm{C})$ & -1.16 & -1.10 & -1.16 & \\
\hline & & $2 s 3 p^{3} \mathrm{P}_{2} 0$ & $58,907.83$ & 989.595 & 0.106 & 7.45 & $8.23(\mathrm{C})$ & & -0.96 & $-0.92(\mathrm{C})$ & -0.94 & -0.87 & -0.94 & \\
\hline & & $2 \mathrm{~s} 4 \mathrm{p}^{3} \mathrm{P}_{0}{ }^{\circ}$ & $66,811.88$ & 4547.688 & 0.014 & 1.01 & $1.12(\mathrm{D})$ & & -0.51 & $-0.46(\mathrm{D})$ & -0.48 & -0.41 & -0.46 & \\
\hline & & $2 \mathrm{~s} 4 \mathrm{p}^{3} \mathrm{P}_{1}{ }^{\circ}$ & $66,811.88$ & 4547.688 & 0.043 & 3.02 & $3.33(\mathrm{C})$ & & -0.03 & $0.01(\mathrm{C})$ & 0 & 0.07 & 0.01 & \\
\hline & & $2 s 4 p^{3} \mathrm{P}_{2}{ }^{\circ}$ & $66,811.88$ & 4547.688 & 0.071 & 5.03 & $5.55(\mathrm{C}+)$ & & 0.19 & $0.24(\mathrm{C}+)$ & 0.24 & 0.29 & 0.24 & \\
\hline \multirow[t]{12}{*}{$2 s 5 d{ }^{3} D_{1}$} & $70,603.76$ & $2 \mathrm{~s} 2 \mathrm{p}^{3} \mathrm{P}_{0}{ }^{\circ}$ & $21,978.28$ & 205.588 & 0.499 & 49.7 & 64.3(B) & & -1.50 & $-1.39(\mathrm{~B})$ & -1.36 & -1.39 & -1.36 & \\
\hline & $\tau=23.3(10)$ & $2 \mathrm{~s} 2 \mathrm{p}^{3} \mathrm{P}_{1}^{\circ}$ & $21,978.925$ & 205.590 & 0.374 & 37.2 & $48.1(\mathrm{C}+)$ & & -1.63 & $-1.52(\mathrm{C}+)$ & -1.48 & -1.51 & -1.49 & \\
\hline & & $2 \mathrm{~s} 2 \mathrm{p}^{3} \mathrm{P}_{2}{ }^{\circ}$ & $21,981.27$ & 205.600 & 0.025 & 2.48 & $3.20(\mathrm{C})$ & & -2.80 & $-2.69(\mathrm{C})$ & -2.66 & -2.69 & -2.67 & \\
\hline & & $2 \mathrm{~s} 3 \mathrm{p}^{3} \mathrm{P}_{0}{ }^{\circ}$ & $58,907.45$ & 854.736 & 0.046 & 4.56 & $5.90(\mathrm{C})$ & & -1.30 & $-1.19(\mathrm{C})$ & -1.32 & -1.22 & -1.24 & \\
\hline & & $2 \mathrm{~s} 3 \mathrm{p}^{3} \mathrm{P}_{1}{ }^{\circ}$ & $58,907.45$ & 854.736 & 0.034 & 3.42 & $4.42(\mathrm{C})$ & & -1.42 & $-1.31(\mathrm{C})$ & -1.45 & -1.35 & -1.37 & \\
\hline & & $2 \mathrm{~s} 3 \mathrm{p}^{3} \mathrm{P}_{2}{ }^{\circ}$ & $58,907.83$ & 854.763 & 0.0023 & 0.228 & $0.300(\mathrm{E})$ & & -2.60 & $-2.49(\mathrm{E})$ & -2.62 & -2.52 & -2.54 & \\
\hline & & $2 s 4 p^{3} \mathrm{P}_{0}{ }^{\circ}$ & $66,811.88$ & 2636.495 & 0.0071 & 0.704 & $0.910(\mathrm{E})$ & & -1.12 & $-1.02(\mathrm{E})$ & -1.19 & -1.03 & -1.08 & \\
\hline & & $2 \mathrm{~s} 4 \mathrm{p}^{3} \mathrm{P}_{1}{ }^{\circ}$ & $66,811.88$ & 2636.495 & 0.0053 & 0.528 & $0.680(\mathrm{E})$ & & -1.25 & $-1.15(\mathrm{E})$ & -1.31 & -1.16 & -1.20 & \\
\hline & & $2 \mathrm{~s} 4 \mathrm{p}^{3} \mathrm{P}_{2}{ }^{\circ}$ & $66,811.88$ & 2636.495 & 0.0004 & 0.0352 & $0.0455(\mathrm{E})$ & & -2.42 & $-2.32(\mathrm{E})$ & -2.49 & -2.33 & -2.38 & \\
\hline & & $2 s 4 f^{3} F_{2}^{\circ}$ & $68,241.02$ & 4231.220 & 0.0039 & 0.384 & $\begin{array}{l}0.497(\mathrm{E}) \\
\text { la }\end{array}$ & & -0.99 & $-0.87(\mathrm{E})$ & -1.02 & -1.08 & -1.04 & \\
\hline & & $2 \mathrm{~s} 5 \mathrm{p}^{3} \mathrm{P}_{0}^{\circ}$ & $70,065.40$ & $18,569.869$ & 0.0015 & 0.148 & $0.192(\mathrm{E})$ & & -0.12 & $-0.004(\mathrm{E})$ & 0.03 & 0.05 & 0.03 & \\
\hline & & $2 s 5 p^{3} \mathrm{P}_{1}^{\circ}$ & $70,065.40$ & $18,569.869$ & 0.0011 & 0.111 & $0.144(\mathrm{E})$ & & -0.24 & $-0.13(\mathrm{E})$ & -0.09 & -0.08 & -0.09 & \\
\hline \multirow[t]{6}{*}{$2 \mathrm{~s} 5 \mathrm{~d}^{1}{ }^{1} \mathrm{D}_{2}$} & $71,002.34$ & $2 \mathrm{~s} 2 \mathrm{p}{ }^{1} \mathrm{P}_{1}^{\circ}$ & $42,565.35$ & 351.554 & 0.962 & 197 & $137(\mathrm{~B}+)$ & & -0.44 & $-0.60(\mathrm{~B}+)$ & -0.77 & -0.74 & -0.60 & $-0.32^{\mathrm{k}}$ \\
\hline & $\tau=35.1(18)^{g}$ & $2 \mathrm{~s} 3 \mathrm{p}^{1} \mathrm{P}_{1}{ }^{\circ}$ & $60,187.34$ & 924.388 & 0.018 & 3.78 & 2.63(E) & & -1.31 & $-1.47(\mathrm{E})$ & -1.28 & -0.91 & -1.22 & \\
\hline & & $2 \mathrm{~s} 4 \mathrm{p}^{1} \mathrm{P}_{1}^{\circ}$ & $67,034.70$ & 2519.702 & 0.003 & 0.594 & $0.413(\mathrm{E})$ & & -1.25 & $-1.41(\mathrm{E})$ & -2.09 & -2.12 & -2.71 & \\
\hline & & $2 \mathrm{~s} 4 \mathrm{f}^{1} \mathrm{~F}_{3}{ }^{\circ}$ & $68,241.18$ & 3620.679 & 0.0002 & 0.0463 & $0.0322(\mathrm{E})$ & & -2.04 & $-2.20(\mathrm{E})$ & & & & \\
\hline & & $2 \mathrm{~s} 5 \mathrm{p}{ }^{1} \mathrm{P}_{1}^{\circ}$ & $70,120.49$ & $11,336.706$ & 0.015 & 3.07 & $2.14(\mathrm{D}+)$ & & 0.77 & $0.61(\mathrm{D}+)$ & 0.69 & 0.70 & 0.67 & \\
\hline & & $2 \mathrm{~s} 5 \mathrm{f}^{1} \mathrm{~F}_{3}{ }^{\circ}$ & $70,749.90$ & $39,602.577$ & 0.0012 & 0.244 & $0.170(\mathrm{E})$ & & 0.76 & $0.60(\mathrm{E})$ & & & & \\
\hline
\end{tabular}


Table 2 (continued)

\begin{tabular}{|c|c|c|c|c|c|c|c|c|c|c|c|c|c|c|}
\hline \multicolumn{2}{|c|}{ Upper level $^{\mathrm{a}}$} & \multicolumn{2}{|c|}{ Lower level $^{\mathrm{a}}$} & \multirow[t]{3}{*}{$\lambda_{\text {air }}(\mathrm{nm})$} & \multirow[t]{3}{*}{$\mathrm{BF}$} & \multicolumn{3}{|c|}{$g A\left(10^{6} \mathrm{~s}^{-1}\right)$} & \multicolumn{6}{|l|}{$\log (g f)$} \\
\hline \multirow[t]{2}{*}{ Assign. } & \multirow{2}{*}{$\begin{array}{l}E\left(\mathrm{~cm}^{-1}\right) \\
\text { Lifetime (ns) }\end{array}$} & \multirow[t]{2}{*}{ Assign. } & \multirow[t]{2}{*}{$E\left(\mathrm{~cm}^{-1}\right)$} & & & \multicolumn{2}{|c|}{ This work } & \multirow{2}{*}{$\begin{array}{l}\text { Previous } \\
\text { Exp. }\end{array}$} & \multicolumn{2}{|c|}{ This work } & \multicolumn{4}{|l|}{ Previous $^{\mathrm{b}}$} \\
\hline & & & & & & HFR & Adjusted $^{\mathrm{n}}$ & & HFR & Adjusted $^{\mathrm{n}}$ & WBEPMT & QDO & MCHF & Exp. \\
\hline \multirow[t]{12}{*}{$2 s 6 s^{3} \mathrm{~S}_{1}$} & $71,160.52$ & $2 \mathrm{~s} 2 \mathrm{p}^{3} \mathrm{P}_{0}^{\circ}$ & $21,978.28$ & 203.260 & 0.071 & 2.69 & $2.91(\mathrm{C})$ & & -2.78 & $-2.74(\mathrm{C})$ & -2.69 & -2.62 & -2.71 & \\
\hline & $\tau=73.4(27)$ & $2 \mathrm{~s} 2 \mathrm{p}^{3} \mathrm{P}_{1}{ }^{\circ}$ & $21,978.925$ & 203.263 & 0.214 & 8.08 & $8.74(\mathrm{C})$ & & -2.30 & $-2.27(\mathrm{C})$ & -2.22 & -2.14 & -2.23 & \\
\hline & & $2 \mathrm{~s} 2 \mathrm{p}^{3} \mathrm{P}_{2}{ }^{\circ}$ & $21,981.27$ & 203.272 & 0.357 & 13.5 & $14.6(C)$ & & -2.08 & $-2.04(\mathrm{C})$ & -1.99 & -1.92 & -2.01 & \\
\hline & & $2 \mathrm{~s} 3 \mathrm{p}^{3} \mathrm{P}_{0}{ }^{\circ}$ & $58,907.45$ & 815.898 & 0.020 & 0.742 & $0.802(\mathrm{D}+)$ & & -2.13 & $-2.10(\mathrm{D}+)$ & -2.09 & -2.05 & -2.10 & \\
\hline & & $2 \mathrm{~s} 3 \mathrm{p}^{3} \mathrm{P}_{1}{ }^{\circ}$ & $58,907.45$ & 815.898 & 0.059 & 2.23 & $2.41(\mathrm{C}+)$ & & -1.65 & $-1.62(C+)$ & -1.62 & -1.58 & -1.63 & \\
\hline & & $2 \mathrm{~s} 3 \mathrm{p}^{3} \mathrm{P}_{2}{ }^{\circ}$ & $58,907.83$ & 815.923 & 0.098 & 3.71 & $4.01(\mathrm{C}+)$ & & -1.43 & $-1.40(\mathrm{C}+)$ & -1.40 & -1.35 & -1.40 & \\
\hline & & $2 \mathrm{~s} 4 \mathrm{p}^{3} \mathrm{P}_{0}{ }^{\circ}$ & $66,811.88$ & 2298.942 & 0.011 & 0.414 & $0.447(\mathrm{E})$ & & -1.48 & $-1.45(\mathrm{E})$ & -1.45 & -1.42 & -1.47 & \\
\hline & & $2 s 4 \mathrm{p}^{3} \mathrm{P}_{1}{ }^{\circ}$ & $66,811.88$ & 2298.942 & 0.033 & 1.24 & $1.34(\mathrm{C})$ & & -1.01 & $-0.97(\mathrm{C})$ & -0.97 & -0.94 & -0.99 & \\
\hline & & $2 \mathrm{~s} 4 \mathrm{p}^{3} \mathrm{P}_{2}{ }^{\circ}$ & $66,811.88$ & 2298.942 & 0.055 & 2.07 & $2.24(\mathrm{C}+)$ & & -0.79 & $-0.75(\mathrm{C}+)$ & -0.75 & -0.72 & -0.77 & \\
\hline & & $2 \mathrm{~s} 5 \mathrm{p}^{3} \mathrm{P}_{0}{ }^{\circ}$ & $70,065.40$ & 9128.931 & 0.009 & 0.345 & $0.370(\mathrm{E})$ & & -0.37 & $-0.33(\mathrm{E})$ & -0.33 & -0.34 & -0.33 & \\
\hline & & $2 \mathrm{~s} 5 \mathrm{p}^{3} \mathrm{P}_{1}{ }^{\circ}$ & $70,065.40$ & 9128.931 & 0.028 & 1.04 & $1.12(\mathrm{C})$ & & 0.11 & $0.15(C)$ & 0.15 & 0.14 & 0.15 & \\
\hline & & $2 \mathrm{~s} 5 \mathrm{p}^{3} \mathrm{P}_{2}{ }^{\circ}$ & $70,065.40$ & 9128.931 & 0.046 & 1.73 & $1.87(\mathrm{C})$ & & 0.33 & $0.39(C)$ & 0.37 & 0.36 & 0.37 & \\
\hline \multirow{8}{*}{$2 \mathrm{~s} 6 \mathrm{~d}{ }^{1} \mathrm{D}_{2}$} & $72,251.27$ & $2 \mathrm{~s} 2 \mathrm{p}^{1} \mathrm{P}_{1}^{\circ}$ & $42,565.35$ & 336.763 & 0.955 & 130 & 83.2(B+) & & -0.66 & $-0.85(\mathrm{~B}+)$ & -1.05 & -0.96 & -0.86 & -0.68 \\
\hline & $\tau=57.3(29)^{\mathrm{g}}$ & $2 \mathrm{~s} 3 \mathrm{p}^{1} \mathrm{P}_{1}{ }^{\circ}$ & $60,187.34$ & 828.689 & 0.030 & 4.02 & $2.58(\mathrm{E})$ & & -1.39 & $-1.58(\mathrm{E})$ & -1.42 & -1.14 & -1.39 & \\
\hline & & $2 s 4 \mathrm{p}^{1} \mathrm{P}_{1}^{\circ}$ & $67,034.70$ & 1916.445 & 0.0002 & 0.0292 & $0.019(\mathrm{E})$ & & -2.79 & $-2.99(\mathrm{E})$ & -2.47 & -1.89 & -2.28 & \\
\hline & & $2 s 4 f^{1} F_{3}{ }^{\circ}$ & $68,241.18$ & 2493.029 & 0.0002 & 0.0277 & $0.018(\mathrm{E})$ & & -2.59 & $-2.78(\mathrm{E})$ & & & & \\
\hline & & $2 \mathrm{~s} 5 \mathrm{p}^{1} \mathrm{P}_{1}{ }^{\circ}$ & $70,120.49$ & 4691.838 & 0.0048 & 0.648 & $0.415(\mathrm{E})$ & & -0.67 & $-0.86(\mathrm{E})$ & -1.30 & -1.13 & -1.46 & \\
\hline & & $2 \mathrm{~s} 5 \mathrm{f}^{1} \mathrm{~F}_{3}{ }^{\circ}$ & $70,749.90$ & 6658.768 & 0.0003 & 0.0396 & $0.0253(\mathrm{E})$ & & -1.58 & $-1.77(\mathrm{E})$ & & & & \\
\hline & & $2 s 6 \mathrm{p}^{1} \mathrm{P}_{1}{ }^{\circ}$ & $71,746.09$ & $19,789.530$ & 0.0095 & 1.29 & $0.827(\mathrm{E})$ & & 0.88 & $0.69(\mathrm{E})$ & 0.76 & 0.79 & 0.76 & \\
\hline & & $2 \mathrm{~s} 6 \mathrm{f}^{1} \mathrm{~F}_{3}{ }^{\circ}$ & $72,111.62$ & $71,588.075$ & 0.001 & 0.134 & $0.086(\mathrm{E})$ & & 1.01 & $0.82(\mathrm{E})$ & & & & \\
\hline
\end{tabular}

a Energy level data are from Kramida and Martin [24], the lifetime data without reference were measured in this paper.

b WBEPMT and QDO are the calculated results from [1] while MCHF are the calculated ones from [29].

c Schnabel and Kock [14].

d Kerkhoff et al. [11].

e Andersen et al. [5].

${ }^{\mathrm{f}}$ Bromander [6].

g Moccia and Spizzo [26].

h Bergström et al. [3]

i Irving et al. [13].

k Andersen et al [7]

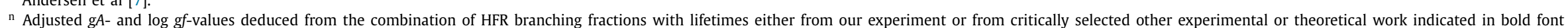
in Table 1. The estimated uncertainties are given in parentheses by code letters used in the NIST database [25], i.e. B $+(\leq 7 \%), \mathrm{B}(\leq 10 \%), \mathrm{C}+(\leq 18 \%), \mathrm{C}(\leq 25 \%)$, D $+(\leq 40 \%), \mathrm{D}(\leq 50 \%)$ and $\mathrm{E}(>50 \%)($ see text). 


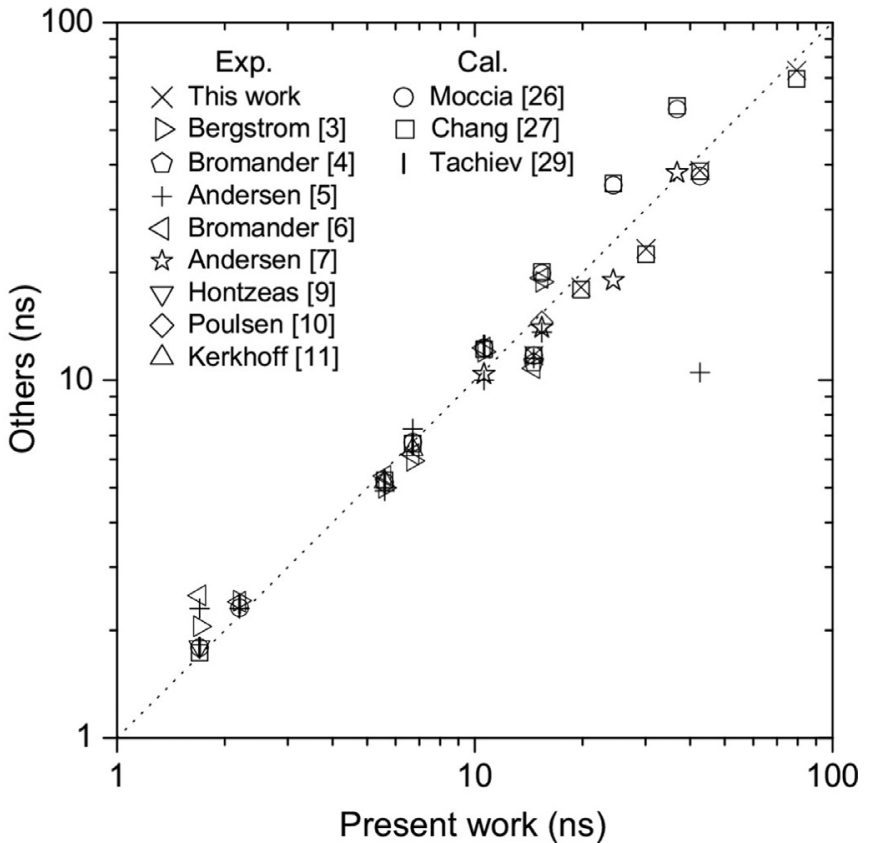

Fig. 3. Comparison between our calculated lifetimes and other experimental and theoretical results. The diagonal dotted line represents equality.

theory by Ateş et al. [1] and with the multiconfiguration HartreeFock (MCHF) theory by Tachiev \& Froese Fischer [29] respectively are also shown in the table for comparisons. For gf values, their differences from our HFR results ((previous - ours)/ours) are below $\pm 20 \%$ for 75,47 and $77 \%$ of the transitions, respectively, and they are less than $\pm 50 \%$ for 92,92 and $95 \%$ of the lines.

To fully utilize the experimental lifetime results, the adjusted $g A$ and $\log (g f)$ values listed in Table 2 were deduced by combining theoretical BFs obtained in this work with measured lifetimes. For comparison, Table 2 also gives $\log (g f)$ values from previous experiments and two calculations. In Table 1 , the lifetime values used for determining the adjusted $g A$ values are marked in bold. From the table one can see that for most of levels previous experimental lifetimes are discrepant, while previous theoretical results are in very good accordance. On the other hand, the eight measured lifetime results from this work and Ref. [11] using selective excitation method, which is free of cascade effect, agree rather well with these theoretical results. This obviously indicates that previous theoretical results are of very high quality and reliability. Therefore, the fact that the radiative parameters of Be I in NIST database [25] take theoretical $g A$ values is understandable. So the lifetimes for other seven levels measured by non-selective excitation were selected based on the good agreement with theoretical results. For the two levels at $71,002.34$ and $72,251.27 \mathrm{~cm}^{-1}$, the experimental lifetimes measured with the beam-foil technique by Andersen et al. [7] strongly deviate from the calculated values, so their experimental results were disregarded and the lifetimes calculated by Moccia and Spizzo [26] were used for deducing the adjusted $g A$ and $\log (g f)$. We used the upper-bound estimated values for the two levels from Ref. [26] and the less-than signs with them are not presented in Table 1 because according to our calculations (see Table 2) the decay branches omitted by the authors have negligibly small contributions to the lifetimes. The uncertainties of the calculated lifetimes from Moccia and Spizzo [26] were evaluated as $3.5 \%$ by comparison with the results deduced from $g A$ values collected in the NIST database [25] for the 15 levels listed in Table 1.

The estimated uncertainties of these adjusted $g A$ and $\log (g f)$ data are also reported in Table 2, using the same code letters as

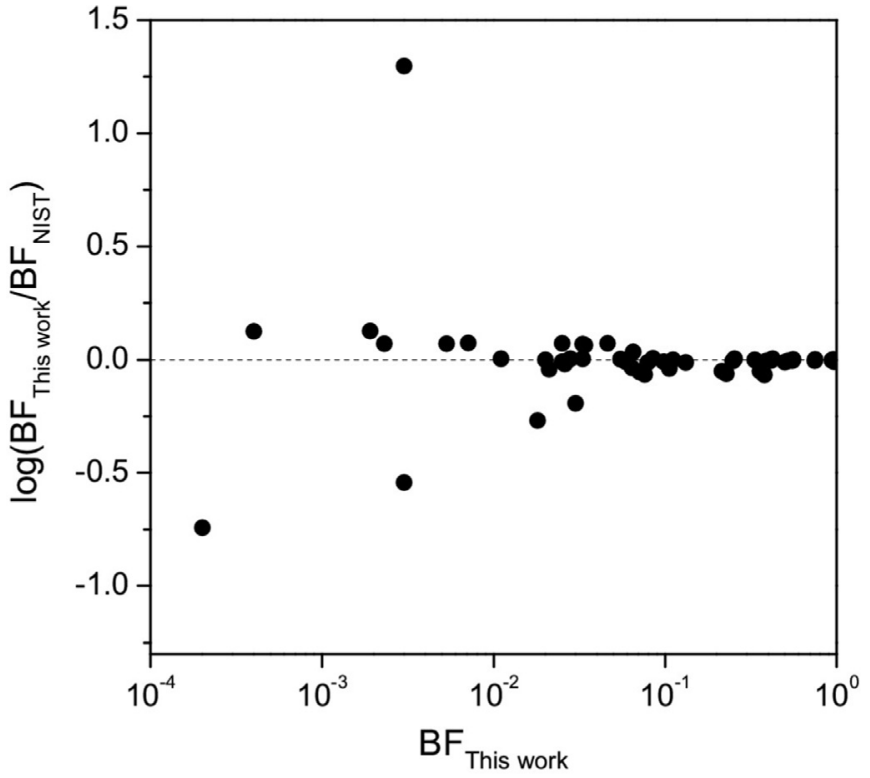

Fig. 4. Comparison between the branching fractions calculated in the present work with those deduced from the transition rates compiled at NIST [25].

those usually employed in the NIST database [25]. They were evaluated as follows. First, an uncertainty was assigned to all our calculated HFR BF-values by comparing the latter to those deduced from the transition rates listed in the NIST database [25]. Such a comparison is shown in Fig. 4 where the ratios $\mathrm{BF}_{\mathrm{This}}$ work $/ \mathrm{BF}_{\mathrm{NIST}}$ are reported against $\mathrm{BF}_{\text {This work }}$. When looking at this figure, one can clearly note a quite regular pattern of increasingly deviating weak branches, the BF-values smaller than 0.005 being affected by large uncertainties (up to an order of magnitude) while larger values have uncertainties in the range $2-30 \%$. These uncertainties were then combined in quadrature with the experimental lifetime uncertainties to yield the uncertainties of $g A$ - and $g f$-values. As a final result, out of the 90 transitions listed in Table 2, there are 16 with an improved accuracy of the decay rates compared to the NIST data, and 7 new transition probabilities that can be assigned an accuracy better than $40 \%$.

Previous experimental $g A$ and $\log (g f)$ results from literature are also presented in Table 2 for comparison. The previously reported experimental $g A$ values are represented by the reciprocals of the measured lifetimes [3,5]. Thus, they correspond to sums of $g A$ values for all possible decay branches of the upper level. They agree well with the sums of our adjusted $g A$ values. As for $\log (g f)$, most levels have larger differences between previous and our adjusted results due to different choices of lifetime data used in the derivation.

\section{Conclusion}

In summary, in this experiment radiative lifetimes for 5 evenparity levels of Be I were measured by the TR-LIF method, and three of them are reported for the first time to our best knowledge. In addition, the HFR + CPOL method was used to calculate transition probabilities and oscillator strengths for 90 Be I lines. Based on the calculated transition probabilities, theoretical branching fractions were deduced to present adjusted transition probabilities and oscillator strengths for these lines by combining the calculated branching fractions with experimental and theoretical lifetimes. The data reported in this paper have been verified by comparison with previous results. 


\section{Acknowledgments}

This work was supported by the National Natural Science Foundation of China (Grant Nos. 11174107 and 11504152), Science and Technology Development Planning Project of Jilin Province (Grant No. 20180101239JC) and Youth Foundation for University of Science and Technology Liaoning (Grant No. 2015QN15). PQ is Research Director of the Belgian F.R.S.-FNRS, financial support from which is gratefully acknowledged.

\section{References}

[1] Ș Ateş, S Özarslan, Celik G, Tașer M. Oscillator strengths for Be I. At Data Nucl Data Tables 2012;98:832-51.

[2] Martinson I. Experimental studies of energy levels and oscillator strengths of highly ionized atoms. In: McDowell MRC, Ferendeci AM, editors. Atomic and molecular processes in controlled thermonuclear fusion. New York: Plenum Press; 1980. p. 391.

[3] Bergström I, Bromander J, Buchta R, Lundin L, Martinson I. Mean lives of excited terms in Be I, Be II, B I, B II, C II and C III. Phys Lett A 1969;28:721-2.

[4] Bromander J, Buchta R, Lundin L. Mean lives of excited terms in beryllium, boron and carbon measured from multiplets in the vacuum ultraviolet region. Phys Lett A 1969;29:523-4.

[5] Andersen T, Jessen KA, Sørensen G. Mean-life measurements of excited electronic states in neutral and ionic species of beryllium and boron. Phys Rev 1969;188:76-81.

[6] Bromander J. Lifetimes and oscillator strengths in spectra of Be, B and C. Phys Scr 1971:4:61-3.

[7] Andersen T, Roberts JR, Sørensen G. Mean-life measurements of excited electronic states in perturbed series of Al II and Be I. Phys Scr 1971;4:52-4.

[8] Berry HG, Bromander J, Martinson I, Buchta R. Doubly-excited terms in lithium and beryllium. Phys Scr 1971;3:63-7.

[9] Hontzeas S, Martinson I, Erman P, Buchta R. Beam-foil studies of energy levels and radiative lifetimes in Be I and Be II. Phys Scr 1972;6:55-60.

[10] Poulsen O, Andersen T, Skouboe NJ. Fast-beam, zero-field level-crossing measurements of radiative lifetimes, fine and hyperfine structures in excited states of ionic and neutral beryllium. J Phys B 1975;8:1393-405.

[11] Kerkhoff H, Schmidt M, Zimmermann P. Lifetime measurements in the 2s3s ${ }^{3} \mathrm{~S}$ and $2 \mathrm{~s} 3 \mathrm{~d}{ }^{3} \mathrm{D}$ states of Be I by selective laser excitation. Phys Lett $\mathrm{A}$ 1980;80:11-13.
[12] Ellis DG, Martinson I, Westerlind M. Spin-induced autoionization of quasibound levels in Be I. Phys Scr 1994;49:561-4.

[13] Irving RE, Henderson M, Curtis LJ, Martinson I, Bengtsson P. Accurate transition probabilities for the $2 \mathrm{~s}^{2}{ }^{1} \mathrm{~S}-2 \mathrm{~s} 2 \mathrm{p}{ }^{1} \mathrm{P}$ transition in Be I and B II. Can J Phys 1999;77:137-43.

[14] Schnabel R, Kock M. f-value measurement of the Be I resonance line using a nonlinear time-resolved laser-induced-fluorescence technique. Phys Rev A 2000;61:062506.

[15] Fuhr JR, Wiese WL. Tables of Atomic Transition Probabilities for Beryllium and Boron. J Phys Chem Ref Data 2010;39:013101.

[16] Kramida A, Fuhr JR. Atomic transition probability bibliographic database. (ver. 9.0); 2017 https://physics.nist.gov/fvalbib.

[17] Tian YS, Wang XH, Yu Q, Li YF, Gao Y, Dai ZW. Radiative lifetime measurements of some Tm I and Tm II levels by time-resolved laser spectroscopy. Mon Not R Astron Soc 2016;457:1393-8.

[18] Zhang W, Palmeri P, Quinet P, Biémont E, Du S, Dai ZW. Radiative-lifetime measurements and calculations of odd-parity highly excited levels in Ba I. Phys Rev A 2010;82:0425071.

[19] Zhang ZG, Li ZS, Svanberg S, Palmeri P, Quinet P, Biémont E. Experimental and theoretical lifetimes in Yb III. Eur Phys J D 2001;15:301-5.

[20] Cowan RD. The theory of atomic structure and spectra. Berkeley: University of California Press; 1981.

[21] Quinet P, Palmeri P, Biémont E, McCurdy MM, Rieger G, Pinnington EH, Wickliffe ME, Lawler JE. Experimental and theoretical lifetimes, branching fractions and oscillator strengths in Lu II. Mon Not R Astron Soc 1999;307:934-40.

[22] Quinet P, Palmeri P, Biémont E, Li ZS, Zhang ZG, Svanberg S. Radiative lifetime measurements and transition probability calculations in lanthanide ions. J Alloys Compd 2002;344:255-9.

[23] Johnson WR, Kolb D, Huang KN. Electric-dipole, quadrupole and magnetic-dipole susceptibilities and shielding factors for closed-shell ions of the He, $\mathrm{Ne}, \mathrm{Ar}, \mathrm{Ni}\left(\mathrm{Cu}^{+}\right), \mathrm{Kr}, \mathrm{Pb}$ and Xe isoelectronic sequences. At Data Nucl Data Tables $1983 ; 28: 333-40$

[24] Kramida A, Martin WC. A compilation of energy levels and wavelengths for the spectrum of neutral beryllium (Be I). J Phys Chem Ref Data 1997;26:1185-94.

[25] Kramida A, Yu Ralchenko, Reader JNIST ASD Team. NIST atomic spectra database (ver. 5.5.1); 2017 https://physics.nist.gov/asd.

[26] Moccia R, Spizzo P. One-photon transition probabilities and photoionisation cross section calculations of Be. J Phys B 1985:18:3537-54.

[27] Chang TN. Radiative lifetimes of the bound excited states of magnesium and beryllium. Phys Rev A 1990;41:4922-9.

[28] Peck ER, Reeder K. Dispersion of Air. J Opt Soc Am 1972;62:958-62.

[29] Tachiev G, Froese Fischer C. Breit-Pauli energy levels, lifetimes, and transition data: beryllium-like spectra. J Phys B 1999;32:5805-23. 